\title{
Scanning photocurrent microscopy of 3D printed light trapping structures in dye-sensitized solar cells
}

\author{
Andrew Knott ${ }^{1}$, Oleg Makarovskiy ${ }^{1}$, James O'Shea ${ }^{1}$, Yupeng $\mathrm{Wu}^{2}$ and Chris Tuck ${ }^{2}$
}

1. School of Physics and Astronomy, The University of Nottingham, NG7 2RD, UK

2. Faculty of Engineering, The University of Nottingham, NG7 2RD, UK

Corresponding author: Andrew Knott ppxak2@nottingham.ac.uk, Chris Tuck Christopher.Tuck@nottingham.ac.uk

\begin{abstract}
Converting solar energy directly into electricity as a clean and renewable energy resource is immensely important to solving the energy crisis and environmental pollution problems induced by the consumption of fossil fuels. Dye-sensitized solar cells (DSSCs) provide a technically and economically credible alternative that could challenge the dominance of conventional $p-n$ junction photovoltaic devices in the solar energy market. DSSCs use dye molecules adsorbed at the surface of nanocrystalline oxide semiconductors such as $\mathrm{TiO}_{2}$ to collect sunlight. These thin films require a large surface area, to adsorb many dye molecules, and mesoporous channels so the electrolyte can permeate the film and regenerate the dye molecules. This favourable morphology is traditionally achieved by the random assembly of a network of nanoparticles by the sintering process.
\end{abstract}

Two-photon polymerization is a 3D printing technique used to fabricate structures with feature resolutions down to $100 \mathrm{~nm}$. We use this technique to fabricate $\mathrm{TiO}_{2}$ thin films of optimised 3D micro-design for use in DSSCs. Our films have a considerable advantage over the conventional (random assembly) films as it allows the implementation of light scattering designs which are shown to significantly enhance photocurrent in the cell by up to $\sim 25 \%$.

\section{Abbreviations:}

DSSC, Dye-sensitized solar cells, TPP, Two-photon polymerization, PGMEA, Propylene glycol methyl ether acetate, FTO Fluorine doped tin oxide.

\section{Keywords:}

Solar energy; Microscopy; Dye-sensitised solar cells; Three-dimensional printing; Twophoton polymerisation 


\section{Introduction}

Scientific and technical advances in photovoltaics over the last few decades have made solar technology one of the most viable alternatives to replace the dominant consumption of fossil fuels for energy production. Dye-sensitized solar cells (DSSCs) have attracted a great deal of attention following their development in 1991 by Grätzel and O'Regan [1]. They provide a technically and economically credible alternative to traditional silicon solar cells due to their low material cost, easy and inexpensive fabrication methods and competitive light to current conversion efficiencies [2]. There are however, challenges facing the development of DSSCs, including the improvement of cell stability [3] and cell efficiencies [4].

A typical DSSC consists of transparent conducting oxide electrode, a mesoporous nanocrystalline oxide semiconductor charge transport layer, photosensitive dye molecules anchored to these nanoparticles to absorb photons, a redox electrolyte to regenerate the dyes and a counter electrode as shown schematically in figure 1. DSSCs typically use thin films of sintered $\mathrm{TiO}_{2}$ nanoparticles for the transport layer [5] since they have a large surface area, required for dye-adsorption, and mesoporous channels, required for electrolyte diffusion.

One area of particular interest for improving efficiency is in the $\mathrm{TiO}_{2}$ thin film. This can be done by increasing the surface area of the film for greater dye absorption, improving the electron transport through the film, and maximising light-harvesting in the layer [6]. In traditional silicon, solar cells many varieties of light management structures in the active layer have been studied. Pillars, pyramids, nanowires have all been shown to increase efficiency in solar cells [7, 8, 9]. However light trapping in DSSCs is more problematic because high performance cells also require the active layer to be nanocrystalline in order to retain a high surface area for dye adsorption and remain porous for dye regeneration by the redox electrolyte.

Many of the top performing DSSCs utilise simple but effective hierarchical structures to enhance light trapping in the active layer in the cell. The typical diameter of $\mathrm{TiO}_{2}$ nanoparticles for dye adsorption and electron transport is $\sim 20 \mathrm{~nm}$ [3], however another light scattering layer of nanoparticles is applied of diameter $\sim 300 \mathrm{~nm}[10]$. This layer has been shown to increase the overall efficiency of the cell by up to $10 \%$ [11]. The light trapping properties of periodic pyramids and other textured surfaces have been well studied in silicon solar cells [12, 13], and more recently studied in DSSCs [14]. More complex light scattering structures in DSSC have been fabricated using a template method whereby a polymer template is fabricated, the template is infiltrated with $\mathrm{TiO}_{2}$ nanoparticles and the template subsequently removed leaving the desired structure behind $[15,16]$. Other interesting and complex light trapping structures 
templated on constructs produced in nature have been incorporated into DSSCs and have shown performance enhancing effects [17]. Labelle et al. have shown from theoretical and experimental work that designed $\mathrm{TiO}_{2}$ pyramids can trap light inside a structure which will internally reflect, increasing the light path length through the active material, increasing the chances of absorbtion. They have shown that a periodic pyramidal patterned film of pitch 10 $\mu \mathrm{m}$, width $8 \mu \mathrm{m}$ and height of approximately $5 \mu \mathrm{m}$ gave an improvement of $24 \%$ in short circuit current in their colloidal quantum dot sensitized solar cells QDSC [18].

There are many interesting and novel methods of introducing light trapping into the active layer of DSSCs to increase cell efficiency. However due to current fabrication methods it is difficult to incorporate design into these structures. In this work, we use a submicron 3D printing technique known as two-photon polymerization (TPP) to fabricate light trapping structures for DSSCs. This technique has the ability to fabricate precise micro and nanostructures with feature resolution of around $150 \mathrm{~nm}$ [19] and has been used for the fabrication of photonic crystals [20,21], and optical interconnects between photonic chips [22]. The utilisation of this technique will allow us to introduce designed light trapping structures into DSSCs. Polymer stamps are fabricated using this technique and then used to pattern $\mathrm{TiO}_{2}$ electrodes. The effectiveness of these structures is then studied using scanning photocurrent microscopy (SPCM) which allows in-depth characterization of the enhancements made by their introduction into the cell. This characterization also allows the study of defects in the cell some of which are invisible using conventional microscope techniques, or when the macroscopic power conversion efficiency of the whole device is measured.

\section{Experimental}

\subsection{Fabrication of polymer stamps}

TPP uses photopolymers such as SU-8 which are traditionally used in 2D photolithography techniques and are irradiated by UV light to drive the polymerization process [23]. However, in TPP absorption occurs when two near-infrared (NIR) photons simultaneously drive the electronic transition. The non-linear dependence of the absorption process allows the excitation to be localized within the volume of the focal point of the NIR-laser, leading to the spatial resolution of $\sim 150 \mathrm{~nm}$, which is better than the diffraction limit of the focussed laser beam [24] as shown schematically in figure 1. Polymer stamps were fabricated using a commercial Nanoscribe system (Photonic Professional, Nanoscribe GmbH, Germany), with a Nanoscribe tailored photoresist IP-L. 
Samples were prepared on glass wafer substrates which were cleaned with acetone, isopropanol and deionized water and subsequently dried under nitrogen gas flow. The commercial Photoresist IP-L (Nanoscribe) was drop cast onto the substrate and the fabrication process began. Negative pyramids were fabricated with dimensions of $8 \mu \mathrm{m}$ width and $5 \mu \mathrm{m}$ depth in 200x200 $\mu \mathrm{m}$ arrays with a $10 \mu \mathrm{m}$ pitch. Once the polymerization process was complete the photoresist was developed in a Propylene glycol methyl ether acetate (PGMEA) solvent bath for 30 mins then removed, bathed a second time in fresh PGEMA, rinsed with isopropanol to remove all un-polymerized material and dried under nitrogen gas flow. Stamps were then characterized (Figure 2a) and checked for quality before being using in electrode fabrication.

\subsection{Preparation of patterned $\mathrm{TiO}_{2}$ electrode}

Fluorine doped tin oxide (FTO) conducting glass substrates (Pilkington, TEC15, sheet resistance $15 \Omega$ /square) were cleaned in an ultrasonic bath with acetone then ethanol and dried under a nitrogen gas flow. Before $\mathrm{TiO}_{2}$ deposition, glass FTO substrates were treated with $\mathrm{TiCl}_{4}$ solution $(0.04 \mathrm{M})$ at $70^{\circ} \mathrm{C}$ for 30 minutes to improve $\mathrm{TiO}_{2}$ adhesion. Substrates were rinsed with deionized water and dried under nitrogen gas flow. $\mathrm{TiO}_{2}$ paste (Ti-Nanoxide, Solaronix, S. A.) was applied to the substrate using a doctor blade technique, one layer of paste was applied and the electrode was dried on a hot plate at $100^{\circ} \mathrm{C}$ for 1 minute. Before the paste was completely dried the stamps were pressed into the paste and left to stand for $5 \mathrm{~min}$ before being removed. The patterned electrodes were then calcinated by slowly heating the samples to $500^{\circ} \mathrm{C}$ at $5^{\circ} \mathrm{C} / \mathrm{min}$ and left for $30 \mathrm{~min}$, the slow heating ramp helps to reduce cracking in the $\mathrm{TiO}_{2}$ film. In some cases, after stamping, the polymer stamps peeled from their glass substrate and remained in the $\mathrm{TiO}_{2}$, the heating process achieves both the sintering of the $\mathrm{TiO}_{2}$ nanoparticles and the removal of these unwanted stamps and any organic material left in the samples. The patterned electrodes were then characterized with SEM and the highest quality selected for cell fabrication. Energy-dispersive X-ray spectroscopy (Hitachi, Table top TM3030) measurements were also made to ensure that no organic material was left on the sample. After characterization, the film was rinsed again in ethanol and dried under a nitrogen gas flow. The electrodes were then sensitized by immersion into $0.5 \mathrm{mM} \mathrm{N}-719$ dye (Solaronix, Ruthenizer 535-bisTBA) ethanol solution for $24 \mathrm{~h}$.

\subsection{DSSCs assembly}

Counter electrodes were prepared by drop casting a Pt solution (Solaronix, Platisol T) onto FTO glass substrates (Pilkington, TEC15, sheet resistance $15 \Omega$ /square) and calcinated at $450^{\circ} \mathrm{C}$ for 15 mins. Sensitized patterned $\mathrm{TiO}_{2}$ electrodes were assembled face to face with 
counter electrodes using $25 \mu \mathrm{m}$ thick thermoplastic frame (Solaronix, Meltonix 1170-25). Finally, the assembled cell was filled with the electrolyte containing the $1 / /^{3-}$ redox system (lodolyte HI-30, Solaronix) through the predrilled hole in the counter electrode, which was then sealed.

\subsection{Scanning photocurrent microscopy of DSSCs}

SPCM uses a focused laser to generate photocurrent locally in a device, scanning therefore allows the mapping of photocurrent response in a large area device. This makes it a powerful non-destructive technique for investigating structures in these devices and how they affect performance. Previously the SPCM technique has been used to investigate optoelectronic properties of a wide variety of semiconductor structures and devices such as nanowire fieldeffect transistors [25], quantum dot thin films [26] and nanowire array solar cells [27]. The SPCM measurements were performed using a focused laser beam (405 nm) onto a sample mounted on an $\mathrm{XY}$-combination of two hybrid piezo-motorised $\mathrm{XY}$-translation stages $\mathrm{M}$ 511. HD (PI Instruments) providing XY-translation up to $10 \mathrm{~cm}$ with precision $<10 \mathrm{~nm}$, electrical current was recorded as a function of the position of the laser spot on the sample.

The SPCM experimental set up is illustrated in Figure $2 a$. Figure $2 b$ and $2 c$ shows the versatility of the system. Spatial resolution of our SPCM set-up can be adjusted between $<1$ $\mu \mathrm{m}$ and $\sim 1 \mathrm{~mm}$ by the diameter of the focussed laser spot. Figure $2 \mathrm{~b}$ shows the entire active area of a conventional $5 \times 5 \mathrm{~mm}$ DSSC cells where the photocurrent is mapped with a resolution of $\sim 300 \mu \mathrm{m}$. In the centre of this map there is clear artefact leading to a corresponding drop in photocurrent, Figure $2 \mathrm{c}$ shows a higher resolution $(\sim 5 \mu \mathrm{m})$ map of this defect. It is worth noting here that this artefact was not visible in optical images of the cell and only became apparent after the cells characterization using SPCM, highlighting the power of this technique as a nondestructive experimental probe for detecting and investigating differences in cells which cannot be detected using conventional optical techniques.

\section{Results and discussion}

Two-photon polymerization allows for the fabrication of repeatable high quality polymer stamps with submicron resolution. Figure 3a shows SEM characterizations of these negative pyramid polymer stamps, here the depth is approximately 5-6 $\mu \mathrm{m}$. Stamps occasionally became dislodged from their glass substrates during post processing, to mitigate these losses stamps were fabricated in batches of 9 separate $200 \mu \mathrm{m}^{2}$ periodic arrays per sample. This allowed for the selection of the highest quality areas of the patterned electrodes during SPCM mapping. These stamps were then used for the patterning of our $\mathrm{TiO}_{2}$ electrodes, Figure $3 \mathrm{~b}$ 
shows a typical SEM image of a good quality portion of the patterned electrodes. Throughout the stamping and calcination process some cracking or deformation of the pyramids is unavoidable. Figure $3 c$ and $3 d$ show a photocurrent map alongside the corresponding optical image of 4 isolated pyramid structures in the centre (and another pyramid to the extreme right of the figure). This map shows a clear enhancement of the photocurrent overlapping with the patterned area of the DSSC, with 4 distinct peaks in photocurrent overlapping the 4 pyramids. The next peak comes over an area where there is damaged pyramid structure. The final peak towards the extreme right is also over a pyramid structure.

Figure $4 \mathrm{a}$ and $\mathrm{c}$ show further enhancement of the photocurrent on patterned areas of the DSSCs. Both maps here show enhancement in photocurrent when compared to the surrounding non-patterned planar $\mathrm{TiO}_{2}$. However, the enhancement in both these photocurrent maps is not uniform across the pattered area. In figure $4 \mathrm{a}$ enhancement in the photocurrent ranges from $\sim 0-5 \%$ in the low enhancement regions and to $\sim 15-20 \%$ in the high enhancement regions. In figure $4 \mathrm{~b}$ enhancement again ranges from $\sim 0-5 \%$ in the low enhancement regions and to $\sim 20-25 \%$ in the high enhancement regions. Figure $4 \mathrm{~b}$ and $\mathrm{d}$ show the corresponding SEM images of these photocurrent maps respectively. These images also show some non-uniformity in stamped pyramid structures, with the areas towards the outside of the array not being as well stamped as the centre. This is reflected in the photocurrent maps where the enhancement is seen in an area less than the $200 \mu^{2}$ patterned region in the SEM images. The images also show some cracking in both samples incurred during calcination, which could help to account for the non-uniformity of the enhancement in the centre of the pattered areas. However, the cracks seen in the corresponding SEM images of figure $4 b$ and $d$ seem to, in some regions, have a positive effect on the photocurrent enhancement and in other regions a negative one.

For more insight into the effects of these cracks on the photocurrent, further defects were probed in different samples, with both patterned areas and planar areas of the cell being characterized. Figure 5a shows the photocurrent map of a large crack that can be seen in the corresponding SEM image in $5 \mathrm{~b}$. Here the crack has a negative effect on the photocurrent leading to a significant drop. This suggests that our SPCM technique provides more accurate local defect characterization than other microscopy techniques. Figure $5 \mathrm{c}$ and $\mathrm{d}$ show the photocurrent map and the corresponding SEM image of another sample of planar $\mathrm{TiO}_{2}$ respectively. Here, although no obvious features are visible in the SEM image there is a clear drop of current in the sample. The drop in current over this small area is significantly larger than the smaller current variations across the rest of this sample. These figures show how effective this technique is at characterising not just visible defects such as cracks and to what 
extent they can affect the cell performance, but also how this technique can be used to find and characterize defects that are hidden below the surface.

Much research has been done into the formation of micro-cracks in crystalline silicon solar cells and how these cracks affect the cells electrical and optical properties [28]. However, it is difficult to get a full picture of how these defects affect cell performance. Cracking in the active layer does not necessarily lead to an initial strong reduction of the cells output power [29] but they do lead to lower cell stability which in turn leads to a reduction of the performance of cell over time [30]. There is also evidence for heavy cracking leading to an introduction of new recombination centres that lead to a subsequent reduction in cell performances [31].

Whist studies of the localisation and characterization of specific cracks and defects in DSSC are rare much care is taken during the fabrication of high performance DSSCs to reduce cracking in the $\mathrm{TiO}_{2}$ to a minimum [32]. When a variety of different $\mathrm{TiO}_{2}$ nanoparticle pastes are studied, pastes which lead to a surface that is heavily populated with micro-cracks has been shown to perform significantly worse than electrodes with smooth surfaces [33]. The bending of $\mathrm{TiO}_{2}$ electrodes in flexible DSSCs tests leads to increased cracking and decreased cell efficiency with increasing number of bends [34], with this reduction being attributed to the decline of the short circuit current from the increased electron transport resistance, and to electron collection. Photocurrent mapping has been used to study intentionally induced defects of cracks on the millimetre scale. These large cracks all lead to significant drops in photocurrent performance [35].

The periodic pyramid patterned $\mathrm{TiO}_{2}$ electrodes fabricated and studied in this paper were able to trap light incident to the cell to enhance the photocurrent generated when compared to planar regions of the sample cell. In this paper, we demonstrate that the 3D-pinting of periodic submicron structures can enhance solar cell performance increasing photocurrent by up to $\sim 25 \%$. This enhancement is in agreement with one observed by Labelle et al. [19] on cells with a similar geometry. Further reports in the literature of DSSCs [36] and QDSCs [37] show the performance-enhancing effects of periodic light trapping microstructures in the $\mathrm{TiO}_{2}$ active layer of these types of solar cells.

\section{Conclusion}

The advantage of our 3D-pinting technique for the fabrication of light trapping structures is the high degree of design that can be incorporated. In this study polymer stamps are fabricated which are used to pattern the $\mathrm{TiO}_{2}$. However, using two-photon polymerization any negative polymer structure, with a resolution approaching the nanoscale, could be produced and the 
$\mathrm{TiO}_{2}$ nanoparticles infiltrated into the template before being removed via calcination much like the removal of the stamps in this study. Highly complex structures with intricate internal 3D light trapping designs can be fabricated using this technique. This can allow designers to no longer be limited by pervious fabrication methods such as the self-assembly of latex spheres [38], or the use of multi-beam interference lithography to make polymer templates [39] which can both create complex internal structures but are restricted in the level of control that can be achieved. This fabrication technique is not limited to the fabrication of light trapping structures in DSSCs and could be employed for the fabrication of structures in a wide variety of photovoltaic devices. Using this technique, a number of theoretically aided light trapping designs can be fabricated onto the same samples a few microns apart thereby reducing the variables involved when trying to compare samples and ensuring almost identical local conditions during characterization. Utilising SPCM these different light trapping structures can be experimentally tested to see which has the best photocurrent performance enhancing effects.

This work demonstrates a flexible and novel 3D-pinting method for the fabrication of DSSC based on periodic submicron structures. Our structures are shown to produce significant performance enhancing effects of up to $\sim 25 \%$ on the photocurrent when compared to planar $\mathrm{TiO}_{2}$. This fabrication technique shows the ability to be able to fabricate highly complex designed 3D light trapping structures that would be impossible using other methods. Furthermore, these structures can be fabricated onto the same sample allowing for close control of conditions across all structures during characterization. This work also shows the effectiveness of the developed SPCM technique in the mapping of local DSSC efficiencies with submicron resolution on a large scale $(>1 \mathrm{~cm})$ devices. Our findings could lead to the development of novel 3D-printed submicron optoelectronic device. 
(a)

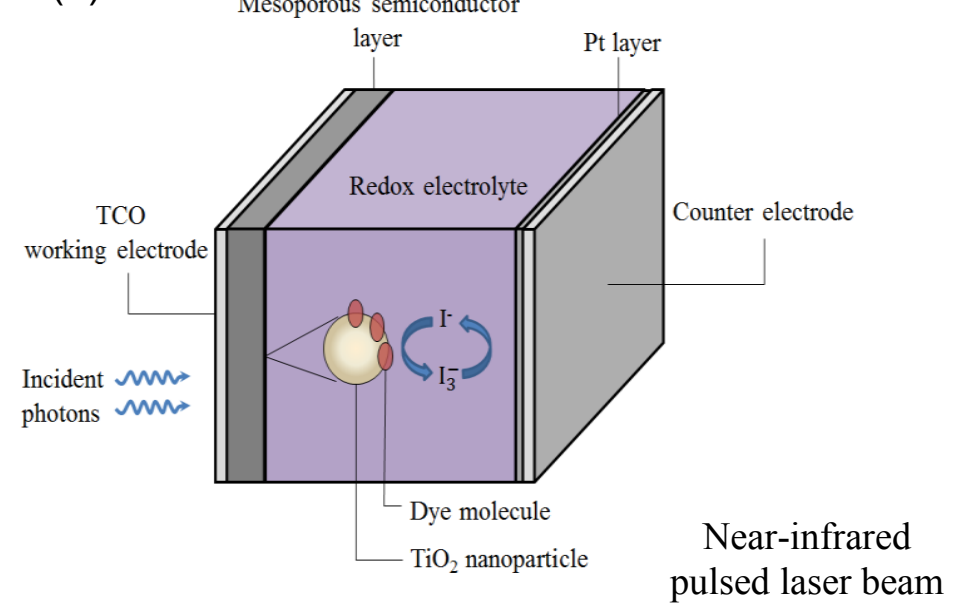

(b)

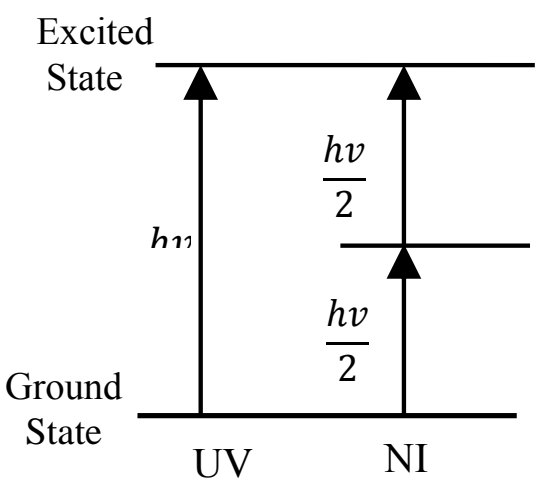

(c)

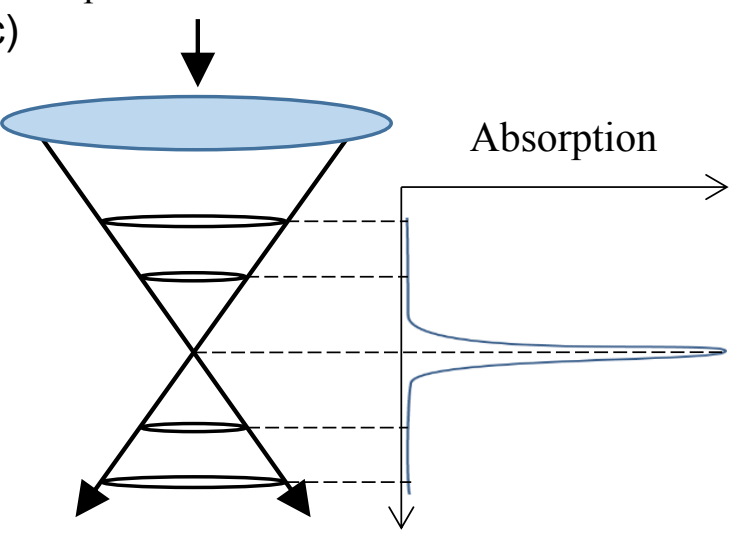

Figure 1 (a) Schematic of DSSCs (b) Single and two-photon absorption processes (c) Absorption of focused laser light in TPP. 
(b)
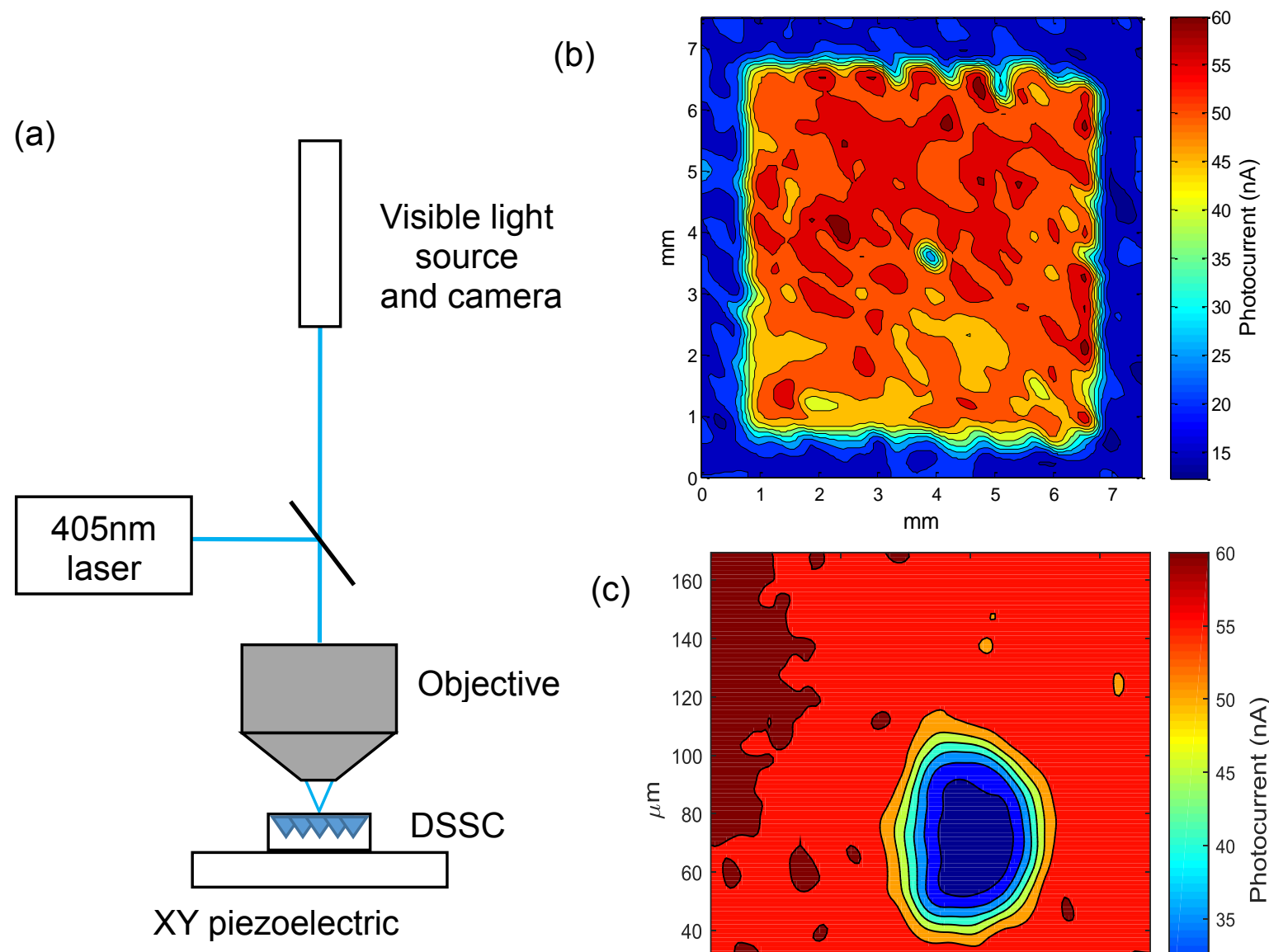

(c)

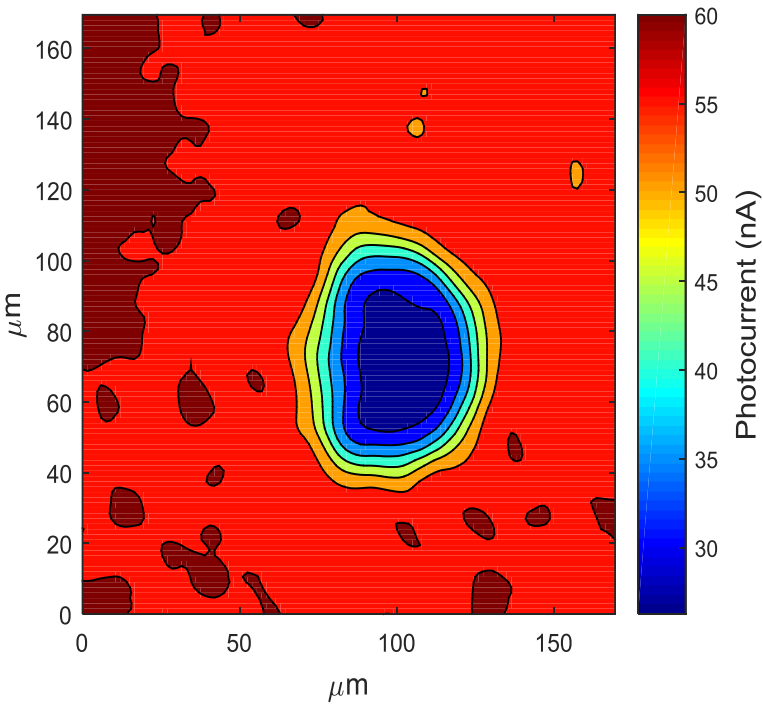

Figure 2 (a) Schematic of experimental set up(b) Low resolution photocurrent map of active area of DSSC (c) High resolution photocurrent map of centre of same cell. 
(a)

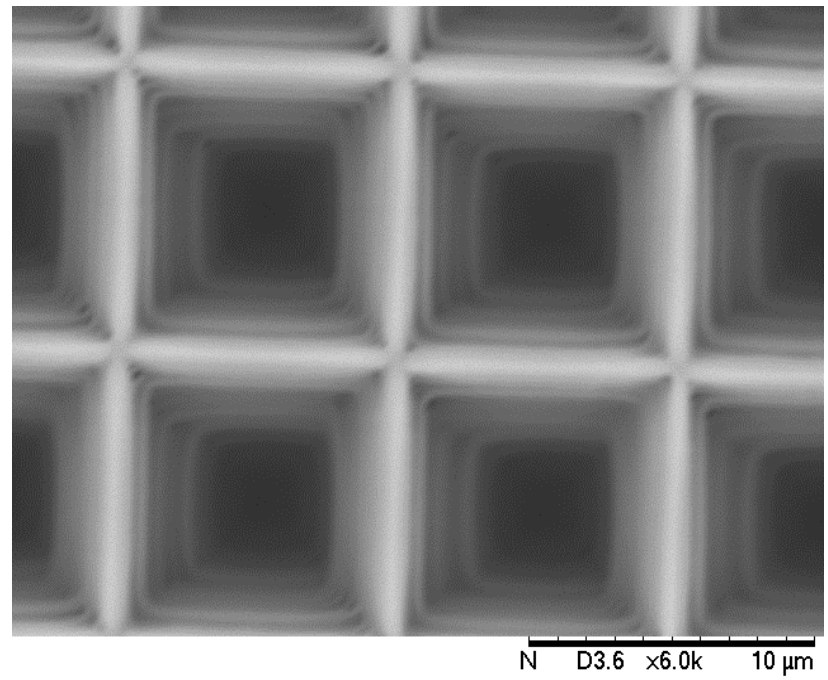

(c)

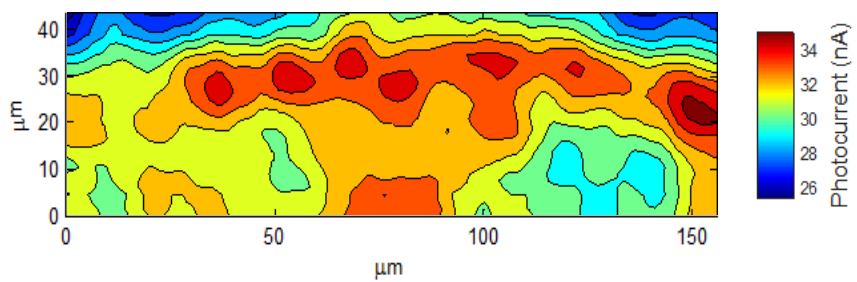

(b)

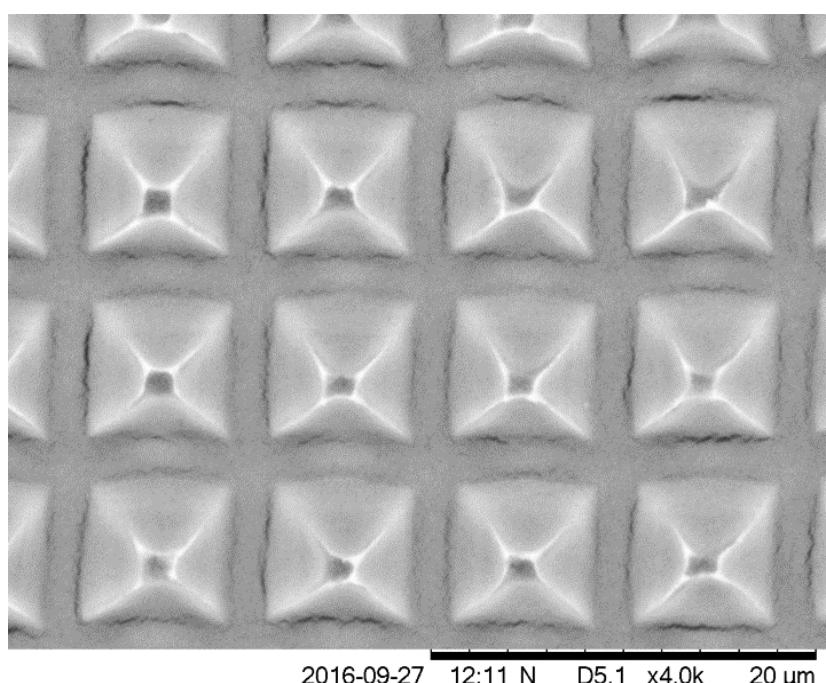

(d)

Figure 3 (a) SEM Image of polymer 3D printed stamps (b) SEM image of corresponding patterned $\mathrm{TiO}_{2}$ electrode (c) Photocurrent map showing enhancement from pyramid structures (d) Corresponding optical image. 
(a)

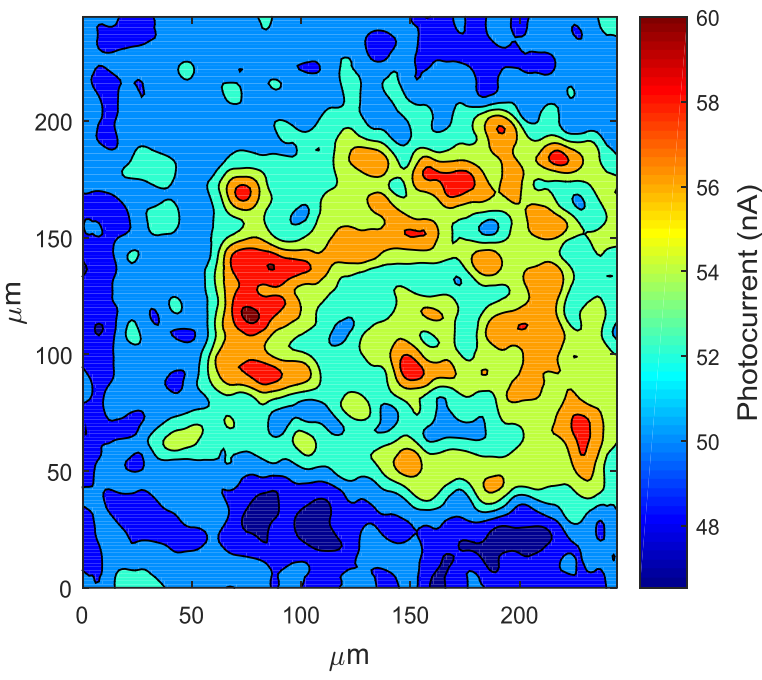

(c)

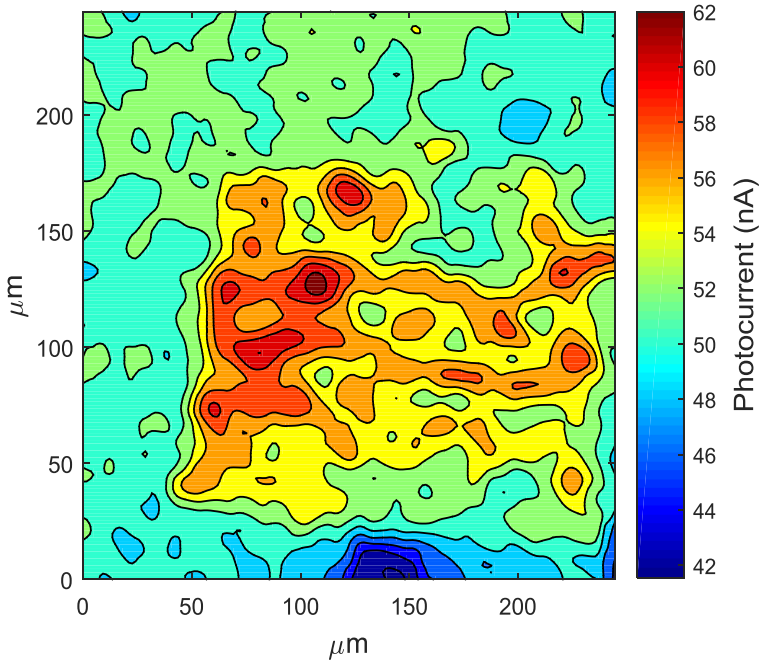

(b)

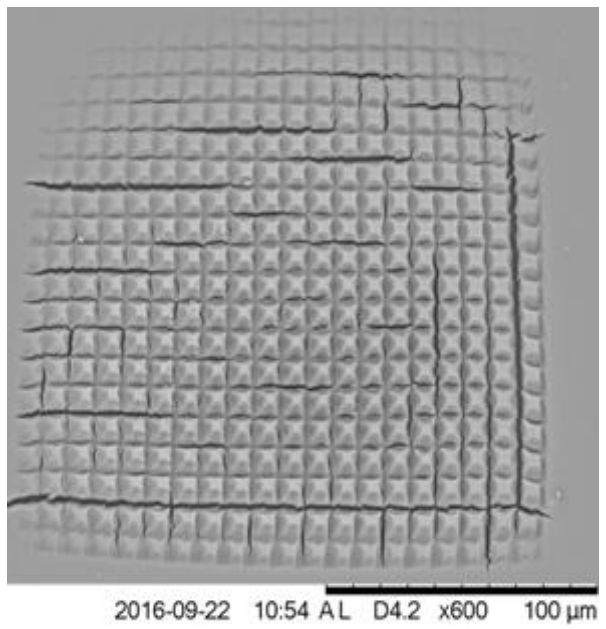

(d)

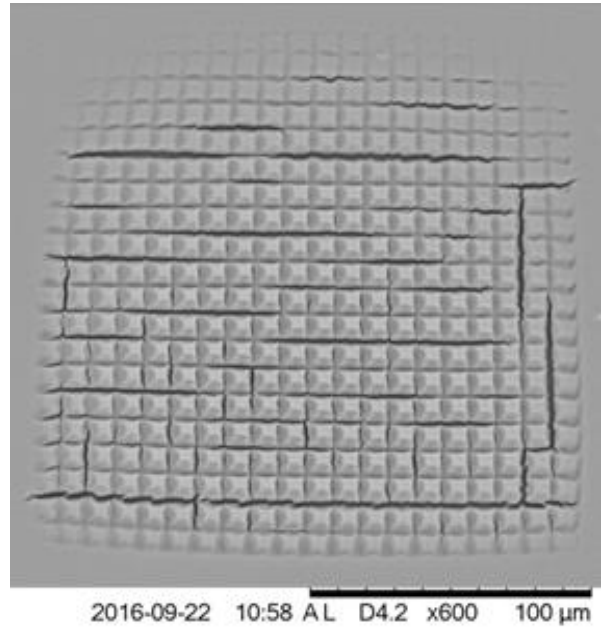

Figure 4 (a) Photocurrent map showing enhancement (b) Corresponding SEM image. (c) Photocurrent map showing enhancement (d) Corresponding SEM images. 
(a)

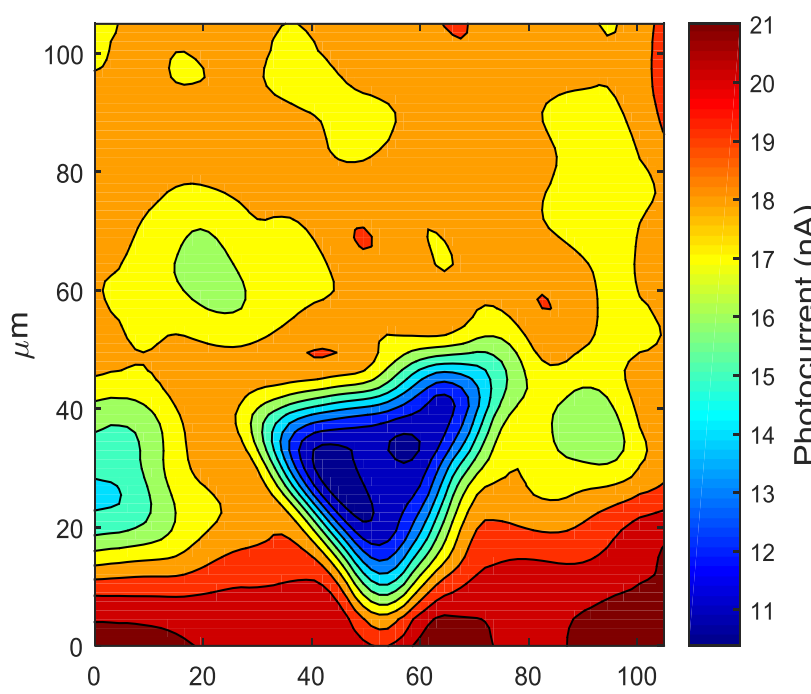

(c)

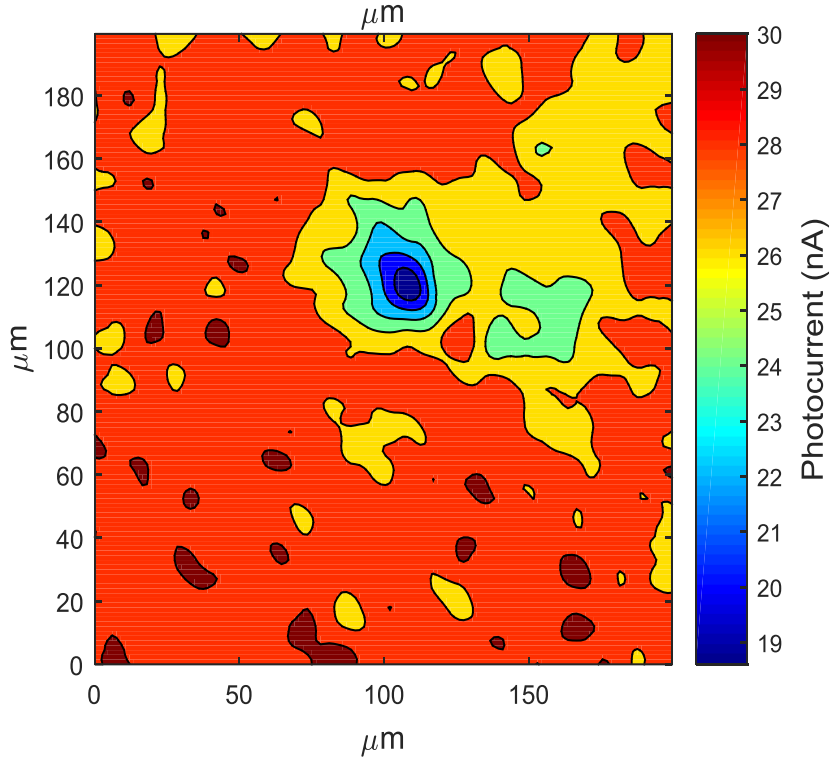

(b)

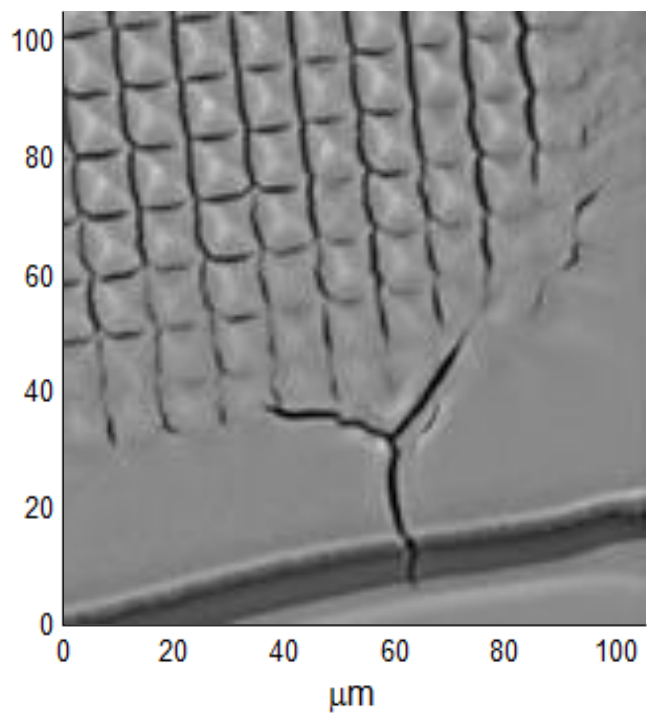

(d)

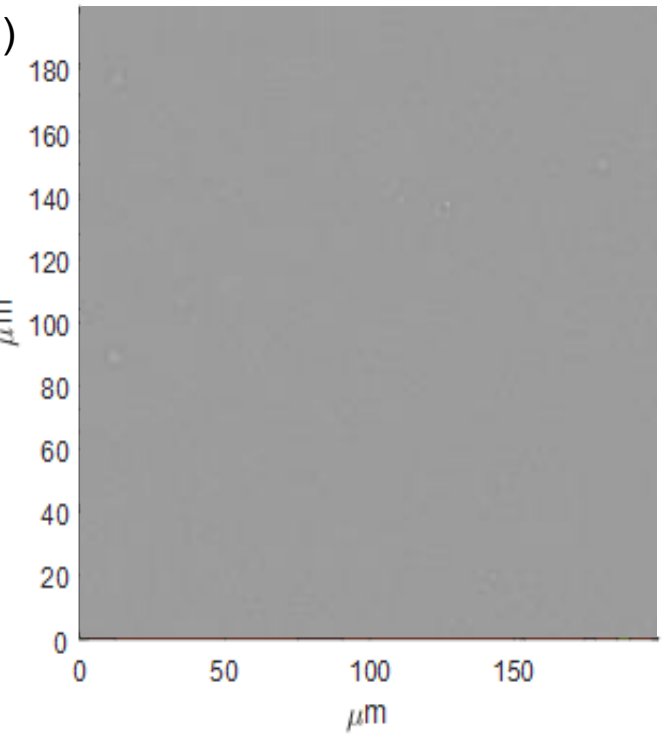

Figure 5 (a) Photocurrent map showing crack in $\mathrm{TiO}_{2}$ electrode (b) Corresponding SEM image (c) Photocurrent map of hidden defect in $\mathrm{TiO}_{2}$ electrode (d) Corresponding SEM image. 


\section{Funding}

This research did not receive any specific grant from funding agencies in the public, commercial, or not-for-profit sectors.

\section{References}

[1] B. O'Regan, M. Grätzel, A low-cost, high-efficiency solar cell based on dye- sensitized colloidal $\mathrm{TiO}_{2}$ films, Nature 353 (1991) 737-793.

[2] M. Grätzel, J. Photochem. C. Photochem. Rev, Dye-sensitized solar cells, 47 (2003) 145153.

[3] A. Hagfeldt, G. Boschloo, L. Sun, L. Kloo, H. Pettersson, Dye-sensitized solar cells, Chem. Rev. 110 (2010) 6595-6663.

[4] S. Zhang, X. Yang, Y. Numata, L. Han, Highly efficient dye-sensitized solar cells: progress and future challenges. Energy Environ. Sci. 6 (2013) 1443-1464.

[5] A. Jena, S. P. Mohanty, P. Kumar, J. Naduvath, and G. Vivekanand, Dye sensitized solar cells: a review, Tans. Ind. Ceram. Soc. 711 (20012) 1-15.

[6] Y. Li, Z-Y. Fu, B-L. Su, Hierarchically structured porous materials for energy conversion and storage, Adv. Funct. Mater. 22 (2012) 4634-4667.

[7] H. P. Yoon, Y. A. Yuwen, C. E. Kendrick, G.D Baber, N. J. Podraza, J. M. Redwing, T. E. Mallouk, C. R. Wronski and T. S. Mayer, Enhanced conversion efficiencies for pillar array solar cells fabricated from crystalline silicon with short minority carrier diffusion lengths, Appl. Phys. Lett. 965 (2010) 213503.

[8] S. J. Jeong, E. C. Garnett, S. Wang, Z. Yu, S. Fan, M. L. Brongersma, M. D. McGehee, Y. Cui, Hybrid silicone nanocone-polymer solar cells, Nano. Lett.12(6) (2012) 2931-2976.

[9] L. Cio, P. Fan, A. P. Vasudev, J. S. White, Z. Yu, W. Cai, J. A. Schuller, S. Fan, M. L. Brongersma, Semiconductor nanowire optical antenna solar absorbers, Nano Lett. 10(2) (2010) 439-445.

[10] S. Hore, C. Vetter, R. Kern, H. Smit, A. Hinsch, Influence of scattering layer on efficiency of dye-sensitized solar cells, Sol. Energ. Mat. Sol. Cells 90 (2006) 1176-1188

[11] S. Ito, T. N. Murakami, P. Comte, P. Liska, C. Grätzel, M. K. Nazeeruddin, M. Grätzel, Fabrication of thin film dye sensitized solar cells with solar electric power conversion efficiency over 10\%, Thin Solid Films 516 (2008) 4613-4619. 
[12] P. Cambell, M. A. Green, Light trapping properties of pyramidally textured surfaces, J. Appl. Phys. 6 (1987) 232.

[13] F. Llopis, I. Tobias, Influence of texture feature size on the optical performance of silicon solar cells, Prog. Photovolt: Res. Appl. 13 (2005) 27-36.

[14] Carmen Lopez-Lopez, S. Colodrero, M. E. Calvo, H. Miguez, Angular response of photonic crystals based dye sensitized solar cells. Energy Environ. Sci. 6 (2013) 1260-1266.

[15] C. Y. Cho, J. H. Moon, Hierarchically porous $\mathrm{TiO}_{2}$ electrodes fabricated by dual templating methods for dye-sensitized solar cells, Adv. Mater. 23, 26 (2011) 2971-2975.

[16] L. Szymanski, P. Surolia, O. Byrne, K. R. Thampi, C. Stubenrauch, Porous "sponge-like" anatase $\mathrm{TiO}_{2}$ via polymer templates: synthesis, characterization, and performance as lightscattering material. Colloid Polym. Sci. 291 (2013) 805-815.

[17] J. Toster, K. S. lyer, W. Xiang, F. Rosei, L. Spiccia, C. L. Raston, Diatom frustules as light traps enhance DSSC efficiency, Nanoscale 5 (2013) 873-876.

[18] A. J. Labelle, S. M. Thon, S. Masala, M. M. Adachi, H. Dong, M. Farahani, A. H. Ip, A. Fratalocchi, E. H. Sargent, Colloidal quantum dot solar cells exploiting hierarchical structuring, Nano. Lett. 15(2) (2015) 1101-1108.

[19] K-S. Lee, R. H. Kim, D-Y. Yang, S. H. Park, Advances in 3D nano/microfabrication using two-photon polymerization, Prog. Polym. Sci. 33 (2008) 631-681.

[20] M. Hermatschweiler, A. Ladermann, G. A. M. Wegener, G. Von Freyman, Fabrication of silicon inverse woodpile photonic crystals, Adv. Funct. Mater. 17 (2007) 2273-2277.

[21] K. Edagawa, S. Kanoko, M. Notomi, Photonic amorphous diamond structure with a 3D photonic band gap, Phys. Lett. Rev. 100(1) (2008) 013901.

[22] N. Lindenmann, G. Balthasar, D. Hillerkuss, R. Schmogrow, M. Jordan, J. Leuthold, W. Freude and C. Koos. Photonic wire bonding: a novel concept for chip scale interconnects, Opt. Express, 2016 (2012) 17667-17677.

[23] M. Miyake, Y-C. Chen, P. V. Braun, and P. Wiltzius, Fabrication of Three-dimensional photonic crystals using multi-beam interference lithography and electrodeposition, Adv. Mater. 21 (2009) 3012-3015. 
[24] L. Li and J. T. Fourkas, Multiphoton Polymerisation, Mater. Today 106 (2007) 30-37.

[25] E. J. Allen, E. R. Hemesath, L. J. Lauhon Scanning photocurrent microscopy analysis of Si nanowire field-effect transistors fabricated by surface etching of the channel, Nano Lett. 9(5) (2009) 1903-1908.

[26] D. P. Ostrowski, M. S. Glaz, B. W. Goodfellow, V. A. Akhavan, M. G. Panthani, B. A. Korgel, D. A. Vanden Bout, Mapping Spatial Hetrogeneity in $\mathrm{Cu}(\ln (1-\mathrm{x}) \mathrm{Ga}(\mathrm{x})) \mathrm{Se} 2$ nanocrystalbased photovoltaics with scanning photocurrent and fluorescence microscopy, Small 6 (2010) 2832-2836.

[27] S. L. Howell, S. Padalkar, K. Yoon, Q. Li, D. D. Koleske, J. J. Wierer, G. T. Wang and L. J. Lauhon. Spatial Mapping of Efficiency of GaN/InGaN, Nanowire array solar cells using scanning photocurrent microscopy, Nano Lett. 13(11) (2013) 5123-5128.

[28] M. Abdelhamid, Review of microcrack detection techniques for silicon solar cells, J. Photovolt. 4(1) (2014) 514-524.

[29] S. Kajari-Schröer, I Kunze, M. Köntges, Criticality of cracks in PV modules, Energy Procedia 27 (2012) 658-663.

[30] M. Köntges, I. Kunze, X. Breitenmoser, B. Bjorenklett, The risk of power loss in crystalline silicone based photovoltaic modules dude to micro-cracks, Sol. Energ. Mat. Sol. Cells 95 (2011) 1131-1137.

[31] J. I. Mölken, U. A. Yusufoğlu, A. Safiei, H. Windgassen, R. Khandelwal, T. M. Pletzer, H. Kurz, Impact of micro-cracks on the degradation of solar cell performance based on two-diode model parameters, Energ. Procedia 27 (2012) 167-172.

[32] X. Wang, M. Xi, F. Zheng, B. Din, H. Fong, Z. Zhu, Reduction of crack formation in $\mathrm{TiO}_{2}$ mesoporous films prepared from binder-free nanoparticles pastes via incorporation of electrospun $\mathrm{SiO}_{2}$ or $\mathrm{TiO}_{2}$ nanofibers for dye-sensitized solar cells, Nano Energy 12 (2015) 794800. 
[33] M. S. Liang, Y. K. Fong, C. C. Khaw, C. C. Liu, S. P. Chin, Studies of Effects of Crystalline Sizes and Scattering Layers on the Conversion Efficient of Dye-Sensitized Solar Cells, Journal of Energy and Power Engineering 2 (2014) 18-24.

[34] X-L. He, G-J. Yang, C-j. Li, M. Liu, S-Q. Fan, Failure mechanism for flexible dye-sensitized solar cells under repeated outward bending: Cracking and spalling of nano-porous titanium dioxide film, J. Power Sources 280 (2015) 182-189.

[35] T. W. Jones, K. Feron, K. F. Andreson, B. C. Duck, G. J. Wilson, An applied lightbeam induced current study of dye-sensitized solar cells: Photocurrent uniformity mapping and true photoactive area evaluation, J. Appl. Phys. 116 (2014) 043104.

[36] F. Yan, J. Kumar, Photovoltaic Performance Enhancement in Dye-Sensitized Solar Cells with Periodic Relief Structure, J. Marcomol. Sci., Pure App. Chem. 46 (2009) 1213-1216.

[37] C. W. Miller, Y. Fu, R. Lopez, Enhancing energy absorption in quantum dot solar cells via periodic light-trapping microstructures, J. Opt., 18(9) (2016) 094002.

[38] L. Qi, D. P. Birnie. Templated titania films with meso- and macroporosities, Matter. Lett. 61 (2007) 2191-2194.

[39] W-M. Jin, J-H. S, C-Y. Cho, J-H. Kang, J. H. Park, J. H. Moon, Holographically defined $\mathrm{TiO}_{2}$ electrodes for dye-sensitized solar cells, ACS Appl. Mater. Interfaces 211 (2010) 29702973. 